\title{
Calidad del sueño en una población adulta expuesta al ruido del Aeropuerto El Dorado, Bogotá, 2012
}

\author{
Lina María Callejas ${ }^{1}$, Rodrigo Sarmiento², Katalina Medina², Henry Sepúlveda,
} Dayana Deluque ${ }^{1}$, Franklin E. Escobar-Córdoba ${ }^{3}$

\author{
1 Línea Aire, Ruido y Radiaciones Electromagnéticas, Hospital Fontibón E.S.E., Bogotá, D.C., Colombia \\ 2 Línea Aire, Ruido y Radiaciones Electromagnéticas, Secretaría Distrital de Salud, Bogotá, D.C., Colombia \\ ${ }^{3}$ Grupo Trastornos del Sueño y Psiquiatría Forense, Universidad Nacional de Colombia, Bogotá, D.C., Colombia
}

Introducción. El Aeropuerto de Bogotá está dentro de la ciudad y su ampliación podría representar un aumento de los efectos adversos del ruido sobre la salud de los habitantes de las localidades de Fontibón y Engativá.

Objetivo. Conocer la prevalencia de los trastornos del sueño y los factores asociados en residentes de Fontibón expuestos al ruido del Aeropuerto El Dorado.

Materiales y métodos. Se hizo un estudio de corte transversal con una muestra de 205 personas de 18 a 65 años de edad, seleccionada mediante muestreo aleatorio estratificado. La calidad del sueño se evaluó mediante el índice de calidad del sueño de Pittsburgh (ICSP) y la escala de somnolencia de Epworth (ESE). Se hicieron análisis estadísticos descriptivos y pruebas de correlación entre estos instrumentos.

Resultados. El $60 \%$ de los residentes informaron mala calidad del sueño según el índice de calidad del sueño de Pittsburgh (ICSP>5), con una media de 7,19 (desviación estándar, DE=3,931), y se encontraron puntuaciones patológicas así: calidad subjetiva del sueño, 27 \%; latencia del sueño, 39 \%; duración del sueño, 33 \%; eficiencia habitual del sueño, 37 \%; alteraciones del sueño, 30 \%; disfunción diurna, $40 \%$, y uso de hipnóticos, $5 \%$. Según la escala de somnolencia de Epworth (ESE>10), se reportó $28 \%$ de somnolencia diurna. Con relación a la prevalencia de la mala calidad del sueño según el índice, $17 \%$ de quienes reportaron no dormir debido al ruido lo asoció al tráfico aéreo. Se encontró correlación entre el índice y la escala $\left(\rho=0,329, I_{95 \%}, 0,20-0,44\right)$.

Conclusiones. Los habitantes de la localidad presentaron mala calidad del sueño por exposición al ruido, siendo la operación aeroportuaria una de las principales fuentes generadoras. Es necesario revisar las estrategias de mitigación de ruido en la localidad y considerar las implicaciones de la ampliación del Aeropuerto El Dorado para la salud pública.

Palabras clave: trastornos del sueño, fases del sueño, contaminación sonora.

doi: http://dx.doi.org/10.7705/biomedica.v35i0.2465

Sleep quality in an adult population exposed to the noise of El Dorado Airport, Bogotá, 2012

Introduction: The airport of Bogotá lies within the city and its expansion could produce an increase in adverse effects on the health of the inhabitants of Fontibón and Engativá districts due to the noise it generates.

Objective: To determine the prevalence of sleep disturbances and associated factors among residents of Fontibón exposed to this noise.

Materials and methods: A cross-sectional study design was used, involving a sample of 205 people aged 18 to 65, selected by means of stratified random sampling. Sleep quality was evaluated using the Pittsburgh Sleep Quality Index (PSQI) and the Epworth Sleepiness Scale (ESS). Descriptive statistics were carried out, as well as correlation tests between the different scales.

Results: A total of $60 \%$ of the residents reported poor quality sleep (PSQI>5), with a mean PSQI of 7.19 (SD=3.931), and the following pathological interruptions were found: subjective sleep quality, 27\%; sleep latency, 39\%; sleep duration, 33\%; habitual sleep efficiency, 37\%; sleep alterations, 30\%; diurnal dysfunction, $40 \%$, and use of sleeping medication, 5\%. According to the Epworth Sleepiness Scale (ESS $>10), 28 \%$ of residents reported daytime somnolence. Regarding the prevalence of poor quality

\section{Contribución de los autores:}

Lina María Callejas, Rodrigo Sarmiento, Katalina Medina, Dayana Deluque y Franklin E. Escobar-Córdoba: primera parte de la elaboración del protocolo y el diseño de la investigación

Lina María Callejas, Katalina Medina, Henry Sepúlveda y Dayana Deluque: coordinación e implementación del trabajo de campo

Todos los autores participaron en el análisis de los resultados. 
sleep according to the Pittsburgh Sleep Quality Index, $17 \%$ of those who reported not being able to sleep because of noise associated this with air traffic. A correlation was observed between the index and the scale ( $\rho=0.329, \mathrm{Cl} 95 \%$ : 0.20-0.44).

Conclusions: Inhabitants of the district reported poor sleep quality due to exposure to noise, airport operations being one of the main generating sources. Noise mitigation strategies in the district need to be reviewed and the public health implications of the El Dorado Airport expansion should be considered.

Key words: Sleep disorders, sleep stages, sound contamination.

doi: http://dx.doi.org/10.7705/biomedica.v35i0.2465

El sueño es un estado fisiológico esencial para la estabilidad y la supervivencia de los seres humanos. La importancia de una buena calidad del sueño es fundamental, no solo como factor determinante de la salud, sino como elemento propiciador de una buena calidad de vida. La calidad del sueño no se refiere únicamente al hecho de dormir bien durante la noche, pues también incluye un buen funcionamiento diurno, es decir, un adecuado nivel de atención para realizar diferentes tareas (1).

El ambiente de descanso determina la duración y la estructura del sueño. El ruido ambiental es uno de los factores más importantes de interrupción del sueño; por su gran incidencia en las ciudades, los ruidos que más se suelen citar en las investigaciones son los producidos por el tráfico, los vecinos y los aviones (2).

La Organización Mundial de la Salud (OMS) considera los efectos del ruido sobre el sueño a partir de los $30 \mathrm{~dB}(\mathrm{~A})$, y sus efectos se clasifican en tres grupos principales: en primer lugar, el ruido puede producir interferencias sobre el mecanismo normal del sueño, ya sea en el momento de conciliarlo o en su transcurso; segundo, alteraciones en la intensidad e interrupciones o despertares, y tercero, efectos que aparecen en la mañana o en el trascurso del día después de la exposición al ruido durante el sueño, los cuales incluyen la reducción en la calidad percibida del sueño, la fatiga, las modificaciones del carácter y las modificaciones en el comportamiento y la sensación de bienestar.

Debido a la localización del Aeropuerto El Dorado dentro del perímetro urbano, Bogotá es una de las ciudades más perjudicadas por la operación aeroportuaria y su población se ha visto afectada por efectos auditivos y 'extraauditivos', que pueden

Correspondencia:

Lina María Callejas, Calle 146 N 7b-77, apartamento 201, Bogotá, D.C., Colombia

Teléfonos: (571) 8108078 y (320) 853 3652; fax: (571) 5432034

lina.callejas@gmail.com

Recibido: 09/07/14; aceptado: 26/03/15 empeorar con la ampliación del terminal aéreo y con su operación durante las 24 horas. Varios estudios han registrado los efectos adversos sobre la salud en las localidades de Fontibón y Engativá, que son las más cercanas al Aeropuerto, pero pocos han evaluado la calidad del sueño de la población afectada $(3,4)$.

El objetivo del estudio fue conocer las posibles relaciones entre la aparición de trastornos del sueño y el ruido en la población residente en el área de influencia del Aeropuerto Internacional EI Dorado en la localidad de Fontibón.

\section{Materiales y métodos}

Se seleccionó el índice de calidad del sueño de Pittsburgh (ICSP), considerado como el instrumento adecuado para establecer la calidad del sueño, ya que es sencillo y fácil de diligenciar y de interpretar, además de que su coeficiente de consistencia interna se encuentra entre 0,78 y 0,81 (alfa de Cronbach), lo cual representa un buen nivel de confiabilidad (5).

EI ICSP es un cuestionario breve, sencillo y bien aceptado, que identifica personas que duermen bien o mal, pero no proporciona un diagnóstico, aunque sus resultados resaltan los aspectos más alterados del sueño (5). El instrumento permite obtener una puntuación global de la calidad del sueño, así como puntuaciones para siete componentes diferentes: calidad subjetiva del sueño, latencia del sueño, duración del sueño, eficiencia habitual del sueño, alteraciones del sueño, uso de medicación hipnótica y disfunción diurna.

También, es importante evaluar las consecuencias diurnas de una mala calidad del sueño, para lo cual se utilizó la escala de somnolencia de Epworth (ESE), instrumento con un alfa de Cronbach de 0,85 . Los dos cuestionarios han sido validados en el español hablado en Colombia (5).

La población objeto incluyó a las personas entre los 18 y 64 años de edad residentes en unidades habitacionales de la localidad de Fontibón que, 
según el censo de población, sumaban 222.681 individuos. Los cálculos se hicieron con el programa Epi-Info 7. Con base en un estudio anterior sobre la percepción del ruido llevado a cabo en dicha población, el tamaño de la muestra se calculó con una prevalencia de $12,75 \%$, una precisión de $5 \%$ y un nivel de confianza de $95 \%$.

La población de la localidad se distribuye en ocho unidades de planificación zonal (UPZ) de la localidad: Fontibón centro (UPZ 75), San Pablo (UPZ 76), Zona Franca (UPZ 77), Ciudad Salitre (UPZ 110), Granjas de Techo (UPZ 112), Modelia (UPZ 114), Capellanía (UPZ 115) y Aeropuerto (UPZ 117). Se seleccionó una muestra al azar con números aleatorios y estratificación con afijación por UPZ, debido a la diferencia en el número de habitantes.

Aunque las zonas de mayor exposición al ruido del Aeropuerto El Dorado son las UPZ 75, 76 y 117, se puede afirmar que en toda la localidad hay un alto grado de exposición al ruido derivado de la operación aeroportuaria.

El análisis estadístico incluyó una fase descriptiva, y pruebas paramétricas y no paramétricas de correlación entre las dos escalas evaluadas mediante el programa estadístico SPSS ${ }^{\mathrm{TM}}$, versión 21.

Los criterios de inclusión de la población de estudio fueron los siguientes: haber residido, al menos, dos años continuos en la localidad; ser mayores de 18 años y menores de 65; residir en el área de influencia del aeropuerto, y querer participar en el estudio. Se excluyó a quienes no eran residentes del lugar y a las personas con alguna limitación física o cognitiva que les impidiera responder la encuesta.

\section{Consideraciones éticas}

Este fue un estudio de bajo riesgo y se apegó a todos los procedimientos establecidos para este tipo de estudios en la Resolución 08430 del Ministerio de Salud. Todas las encuestas se hicieron con previo consentimiento informado por parte de los participantes. La información recolectada pertenece a la Secretaría Distrital de Salud de Bogotá y al Hospital Fontibón E.S.E., y su uso no está autorizado para fines diferentes a los de este trabajo y está sujeta a la preservación del secreto profesional.

\section{Resultados}

Se encontró que 54 (26,34 \%) de las 205 personas encuestadas eran hombres y $151(73,7 \%)$ mujeres.

\section{Puntaje total del índice de calidad del sueño de Pittsburgh}

Con la aplicación de este índice se determinó si la población residente en el área de influencia del Aeropuerto Internacional EI Dorado en la localidad de Fontibón tenía un buen o mal dormir durante los meses de octubre a diciembre del 2012 y enero del 2013.

Se encontró que $40 \%$ (82/205) de los encuestados presentaban puntuaciones totales menores o iguales a 5, puntaje que se considera normal para esta población. Los puntajes mayores de 5 , considerados patológicos, se encontraron en $60 \%(123 / 205)$ de los encuestados. La media de la puntación global fue de 7,19 $(\mathrm{DE}=3,93)$ y la mediana fue de 6,00 (cuadro 1).

\section{Calidad subjetiva, latencia, duración y eficiencia del sueño}

Al explorar la calidad subjetiva del sueño, se encontró que el $72 \%$ (148/205) de los encuestados la calificaron como "muy buena o bastante buena", calificación que se considera normal. La calidad subjetiva del sueño calificada como "bastante mala o muy mala", que se considera patológica, se halló en $28 \%(58 / 205)$ de los encuestados (cuadro 2$)$.

De acuerdo con la definición universal, una latencia inadecuada del sueño se considera como un insomnio de conciliación o inicial de tipo crónico. Al explorar este dominio, se encontró que 125 (61\%) de los encuestados presentaban una latencia normal del sueño. La latencia inadecuada del sueño se halló en 80 (39\%) de los encuestados.

Según la definición aceptada, la duración del sueño en el último mes se considera inadecuada cuando es menor de siete horas por noche. Al explorar este dominio, se encontró que el $67 \%$ (138/205) de los encuestados presentaron una duración adecuada del sueño y en el $33 \%$ (60/205) fue inadecuada (cuadro 2).

Cuadro 1. Puntaje global obtenido en el índice de calidad del sueño de Pittsburgh

\begin{tabular}{lc}
\hline Índice de calidad del sueño de Pittsburgh \\
\hline Media & \\
Mediana & 7,19 \\
Moda & 6,00 \\
Desviación estándar & 4 \\
Varianza & 3,931 \\
Mínimo & 15,449 \\
Máximo & 0 \\
\hline
\end{tabular}

Fuente: Línea Aire, Ruido, REM, Hospital Fontibón, 2013 
Cuadro 2. Calidad del sueño según percepción subjetiva, duración y eficiencia del sueño

\begin{tabular}{lcccccc}
\hline & \multicolumn{2}{c}{ Calidad del sueño } & \multicolumn{2}{c}{ Duración del sueño } & \multicolumn{2}{c}{ Eficiencia del sueño } \\
\cline { 2 - 7 } & \multicolumn{1}{c}{$\mathbf{n}$} & $\%$ & $\mathbf{n}$ & $\%$ & \multicolumn{1}{c}{$\mathbf{n}$} & $\%$ \\
\hline Muy buena & 32 & 15,6 & 67 & 32,7 & 129 & 62,9 \\
Bastante buena & 116 & 56,6 & 67 & 32,7 & 34 & 16,6 \\
Bastante mala & 42 & 20,5 & 43 & 21 & 16 & 7,8 \\
Muy mala & 15 & 7,3 & 28 & 13,7 & 26 & 12,7 \\
Total & 205 & 100 & 205 & 100 & 205 & 100 \\
\hline
\end{tabular}

Fuente: Línea Aire, Ruido, REM, Hospital Fontibón, 2013

De acuerdo con la definición universalmente aceptada, la eficiencia del sueño, la cual se calcula como el número total de horas dormidas en el último mes dividido por el número total de horas en cama multiplicado por 100 , se considera inadecuada cuando el resultado es menor de $85 \%$. Al explorar este dominio, se encontró que en 129 (63\%) de los encuestados era adecuada y en 76 (37\%) era patológica (cuadro 2).

\section{Puntaje total de perturbaciones del sueño}

De acuerdo con la calificación del ICSP, el puntaje total de perturbaciones del sueño se considera inadecuado cuando es mayor de 10 puntos. Al explorar este dominio, se halló que el $70 \%$ (143/205) de los encuestados presentaba un puntaje total no mayor de 10 puntos, puntuación considerada como normal. En el $30 \%$ (62/205) de los encuestados se halló un puntaje total mayor de 10 puntos, el cual se considera patológico.

Mediante los componentes del ICSP, se estimaron las variables relativas a "tener problemas para dormir por despertarse durante la noche o de madrugada", "tener que levantarse para ir al baño", "no poder respirar bien", "toser o roncar ruidosamente", "sentir frío", "sentir calor", "tener dolores", "tener malos sueños o pesadillas" y "otras razones" durante el mes anterior a la entrevista. De acuerdo con la calificación del ICSP, si la respuesta era "una o más veces a la semana", se consideraba que había un patrón inadecuado del sueño.

En cuanto al dominio de "otras razones", se evidenció que el $63 \%(131 / 205)$ de la población encuestada manifestó que ninguna otra razón le había generado problemas para dormir en el mes anterior y el 23 \% (47/204) mencionó algún tipo de ruido. De la población que manifestó tener problemas para dormir debido al ruido, el $17 \%$ (8/47) especificó que el ruido de los aviones le había impedido conciliar el sueño en el mes anterior, mientras que solo el $4 \%(2 / 47)$ reportó problemas de sueño debidos al tráfico terrestre. Además, $1 \%$ (2/205) de los encuestados manifestó haber sido diagnosticado con apnea del sueño.

\section{Uso de medicación para dormir y disfunción diurna}

De acuerdo con la definición de la escala, se consideró que había consumo de hipnóticos cuando el encuestado respondía que se le habían formulado o los había tomado por su cuenta "más de una vez a la semana" durante el mes anterior. Al explorar este aspecto, se halló que el $95 \%$ (194/205) de los encuestados no había consumido hipnóticos y que el $5 \%$ (11/205) sí lo había hecho.

\section{Calidad de la vigilia}

Como lo define la escala, si la persona respondía haber tenido disfunción una o más veces a la semana, la calidad de la vigilia se consideraba alterada. Al explorar esta pregunta, se halló que el $61 \%(124 / 205)$ de los encuestados no presentaban disfunción diurna y el $40 \%$ (81/205) de ellos, sí (cuadro 3).

\section{Comportamiento sociodemográfico con relación a la calidad del sueño}

Con relación a la puntuación global del ICSP en las UPZ estudiadas, la media en la UPZ 75, Fontibón, fue de $7,75 \pm 4,04$, con una mediana de 7,00 ; en la UPZ 76, San Pablo, fue de 6,79 $\pm 4,41$, con una mediana de 5,00; en la UPZ 77, Zona Franca, fue de $7,61 \pm 4,40$, con una mediana de 7,80 ; en la UPZ 110, Ciudad Salitre, fue de 6,04 $\pm 2,75$, con

Cuadro 3. Calidad de la vigilia

\begin{tabular}{lcc}
\hline & $\mathbf{n}$ & $\%$ \\
\hline Nada problemática & 50 & 24,4 \\
Ligeramente problemática & 74 & 36,1 \\
Moderadamente problemática & 59 & 28,8 \\
Muy problemática & 22 & 10,7 \\
\hline
\end{tabular}

Fuente: Línea Aire, Ruido, REM, Hospital de Fontibón, 2013 
una mediana de 5,50; en la UPZ 112, Granjas de Techo, fue de 6,93 $\pm 3,26$, con una mediana de 6,93; en la UPZ114, Modelia, fue de 5,95 $\pm 4,04$, con una mediana de 6,00 , y en la UPZ 115, Capellania, la media fue de $6,90 \pm 3,84$, con una mediana de 6,00 . En la UPZ 117, Aeropuerto, solo se encuestó a un sujeto, cuya puntuación fue de 5 .

Se evidenció que en todas las UPZ, exceptuando la UPZ 117, las medias correspondieron a puntuaciones por encima de 5, las cuales se consideran patológicas en la escala.

En cuanto a la distribución de los datos por sexo, se encontró que el 63,6 \% (96/151) de las mujeres $y$ el $50,0 \%(27 / 54)$ de los hombres obtuvieron puntajes patológicos.

En cuanto a los grupos de edad, se presentó la siguiente distribución de datos patológicos: de 18 a 30 años, el 58,3 \% (14/24) de los encuestados presentaron puntuaciones patológicas; de 31 a 50 años, el $55,1 \%(54 / 98)$ y de 51 a 64 , el $66,3 \%(55 / 83)$.

\section{Escala de somnolencia de Epworth}

Mediante la aplicación de esta escala se encontró que el $72 \%$ (148/205) de los encuestados presentaron puntuaciones totales menores o iguales a 10, puntaje considerado como normal. Los puntajes mayores de 10, que corresponden a somnolencia diurna excesiva, se presentaron en el $28 \%$ (57/205) de los encuestados (cuadro 4).

Hubo correlación entre las dos escalas tanto en las pruebas paramétricas $\left(\rho=0,329, I_{95 \%} 0,20\right.$ 0,44 ), como en las no paramétricas (coeficiente de Spearman $=0,27$ ), las cuales fueron significativas $(p<0,05)$.

\section{Discusión}

Este es uno de los primeros estudios en que se examina la prevalencia de los trastornos del sueño relacionados con el posible impacto del

Cuadro 4. Puntuación global en la escala de somnolencia de Epworth

\begin{tabular}{lrc}
\hline Puntaje & $\mathbf{n}$ & $\%$ \\
\hline$<7$ & 102 & 49,8 \\
$8-9$ & 38 & 18,5 \\
$10-15$ & 41 & 20,0 \\
$>16$ & 24 & 11,7 \\
Total & 205 & 100,00 \\
\hline
\end{tabular}

Fuente: Línea Aire, Ruido, REM, Hospital de Fontibón, 2013 ruido procedente del Aeropuerto Internacional EI Dorado, en la población residente en su área de influencia en la localidad Fontibón. Los resultados relativos a las variables estudiadas evidencian frecuencias indicativas de posibles alteraciones de la calidad del sueño en la población participante, con puntuaciones que se consideran patológicas. La muestra fue representativa para la localidad de Fontibón y los hallazgos revelaron aspectos interesantes dignos de discusión.

En un estudio llevado a cabo en Filadelfia en el 2010, Basner, et al. (6), encontraron que los pequeños cambios inducidos por el ruido en la estructura y la continuidad del sueño, eran suficientes para afectar de forma significativa la evaluación subjetiva de la calidad del sueño y la recuperación, lo cual coincide con los resultados del presente estudio en los que alrededor del $25 \%$ de la población encuestada expresó una percepción subjetiva de mala calidad del sueño, en tanto que cerca del $40 \%$ de la población con puntajes patológicos tuvo una latencia inadecuada, $y$, alrededor de $37 \%$, una eficiencia del sueño baja.

Si el ruido perturba repetidamente el sueño, no se consigue el descanso necesario y al despertar se tiene una cierta perspectiva de la calidad del sueño. El desarrollo natural de la etapa de sueño con movimientos oculares rápidos, tiene el papel más crítico en dicha percepción (7).

En este estudio se encontró que la puntuación total del instrumento (ICSP) en el $60 \%$ de los encuestados superaba los 5 puntos, por lo que se puede considerar a estos sujetos como personas de mal dormir. Es importante tener en cuenta que dicha puntuación podría responder a la exposición al ruido durante la noche, con efectos susceptibles de que pueden medirse al día siguiente. Algunos estudios han demostrado que, cuando el individuo está despierto, su percepción de la calidad del sueño es mala, y reporta un incremento de la fatiga, un estado de ánimo deprimido y un desempeño deficiente, entre otros (2).

Por otro lado, al analizar los distintos dominios del ICSP, se evidenció que el $25 \%$ de las personas encuestadas percibía como inadecuada la calidad del sueño, calificándola como "bastante mala o muy mala", lo que vendría a corroborar el planteamiento de que el ruido percibido conscientemente determina la evaluación subjetiva de la calidad del sueño (6). 
De igual forma, cabe anotar que la percepción subjetiva del sueño fue menor en los sujetos que obtuvieron un puntaje total del ICSP indicativo de mal dormir; aun así, en el dominio de las perturbaciones del sueño se encontró que el 30 $\%$ de los encuestados obtuvieron un puntaje por encima de los 10 puntos, el cual corresponde a manifestaciones patológicas en dicho dominio.

Con relación a la operación del Aeropuerto El Dorado, se observó que del total de personas que manifestaron tener alguna perturbación en el sueño asociada al ruido, el $17 \%$ aducía que el ruido debido al tráfico aéreo repercutía en la calidad de su sueño. La afectación por ruido puede ser más alta, pero estos resultados podrían tener relación con lo planteado por Muzet con respecto a la transición de las etapas de sueño profundo a las de un sueño más ligero, con una asociación directa entre los despertares electroencefalográficos y el ruido nocturno. Estas transiciones no deseadas no son percibidas por el sujeto, pero modifican la arquitectura del sueño y la cantidad total de ondas de sueño lentas y de sueño de movimientos oculares rápidos, produciendo un cambio hacia un sueño superficial en detrimento del sueño profundo (2).

En la comparación de los efectos en el sueño del ruido procedente del tráfico aéreo, terrestre y ferroviario hecha por Basner, et al., se presentaron diferencias en el efecto sobre la estructura del sueño reportado de forma objetiva y subjetiva. Según su estudio, los sujetos encuestados calificaron las noches en las que hubo exposición al ruido del tráfico aéreo como "más preocupantes" que aquellas afectadas por el ruido del tráfico terrestre, por lo que los autores concluyeron que las propiedades acústicas del ruido del tráfico aéreo podrían ser las responsables de los cambios en la estructura y la continuidad del sueño. Es posible que los eventos de ruido generados por el tráfico en carretera fueran demasiado cortos para ser percibidos conscientemente por los sujetos que se despertaron, mientras que los sucesos de ruido debidos al tráfico aéreo pudieron haber sido lo suficientemente largos (6).

Estas consideraciones podrían explicar por qué entre las perturbaciones detectadas mediante la aplicación de los instrumentos de evaluación de la calidad del sueño en la localidad de Fontibón, se encontró que el $44 \%$ de las personas manifestaron haber tenido problemas para conciliar el sueño durante la primera media hora y, el $65 \%$, haberse despertado durante la noche. Estas cifras son muy superiores a las encontradas por Rueda, et al., en el estudio llevado a cabo entre la población general de Bucaramanga, Colombia, en el que hallaron que el $11,4 \%\left(\mathrm{IC}_{95 \%} 9,7-13,1\right)$ de las personas encuestadas dijeron tener problemas de sueño, y que el 63,6\% $\left(\mathrm{IC}_{95 \%} 61,0-66,3\right)$ de ellas referían, al menos, un síntoma de insomnio, pero sin consecuencias al otro día, en tanto que el 26,2 \% (IC $\left.{ }_{95 \%} 23,7-28,7\right)$ manifestó tener, al menos, un síntoma de insomnio con consecuencias durante el día siguiente (8).

Por último, es importante mencionar que en la encuesta de somnolencia el $40 \%$ de las personas encuestadas manifestaron haber experimentado alteraciones en la calidad de la vigilia, reportando problemas para permanecer despiertas durante el día o falta de ánimo para llevar a cabo sus actividades cotidianas de manera apropiada, lo que puede ser consecuencia de un mal descanso nocturno, como quedó manifiesto al indagar sobre la variable de la calidad del sueño percibida subjetivamente, en la que el $25 \%$ de los encuestados manifestaron que esta era mala.

Esta última variable del ICSP se relacionó con la ESE en lo concerniente a la medición del grado sufrido de somnolencia diurna, lo que desempeña un papel importante en el diagnóstico de posibles trastornos del sueño.

Como ya se mencionó, uno de los efectos del ruido es la mala calidad del sueño y el cansancio percibidos después de dormir (somnolencia). Esto se puede explicar por los cambios en las fases del sueño y por el acortamiento del sueño profundo y del sueño de movimientos oculares rápidos y la prolongación de las fases de sueño no profundo. Los puntajes mayores de 10 en la ESE se consideraron equivalentes a la somnolencia diurna excesiva, lo que sugiere un posible trastorno del sueño en la población evaluada en este estudio. De las 205 personas encuestadas, 57 (28\%) obtuvieron puntajes patológicos. Tal como menciona Kawada, las personas que están expuestas al ruido de tráfico terrestre y aéreo durante la noche reportan una mala calidad de sueño y gran irritabilidad durante el transcurso del día. La explicación de la inestabilidad del sueño de movimientos oculares rápidos cuando se está expuesto al ruido, se encuentra en el hecho de que en esta fase aumenta la actividad muscular del oído medio (9), lo que lleva a pensar que el porcentaje de la población de este estudio con puntajes por encima de los 10 puntos podría llegar a tener trastornos en el sueño. 
Se evidenció que el impacto de las perturbaciones en la calidad del sueño es bastante notorio en la localidad de Fontibón, al igual que los puntajes globales obtenidos en el ICSP. Una mala calidad del sueño trae como consecuencia el cansancio y la tendencia al mal humor. Si se duerme "mal" a causa del ruido, durante el día siguiente baja el desempeño en las "tareas psíquicas" y motoras, debido al cansancio y el mal humor (7).

Este estudio presenta una primera aproximación a los efectos del ruido sobre la calidad del sueño, en especial, el generado por la operación aeroportuaria. Las escalas utilizadas se consideran instrumentos costo-efectivos para evaluar la asociación entre la calidad del sueño y el ruido (6). Para confirmar los hallazgos de este estudio, las investigaciones posteriores deberán evaluar la calidad del sueño mediante otros métodos, como la polisomnografía y la actigrafía, sugeridos por algunos autores.

El ruido urbano interfiere en varias actividades de la vida diaria, tornándolas más difíciles de efectuar, sobre todo cuando se necesita concentración, y produce molestias e interferencias en la comprensión del discurso; además, interrumpe el sueño y provoca efectos fisiológicos que degradan la calidad de vida y el bienestar de las personas expuestas a él. Dado que la mayoría de los ruidos provienen del exterior de las viviendas, es prioritario buscar sistemas que los reduzcan, lo que significa invertir a corto y largo plazo en la mejoría del sueño y de la salud de las personas. Así mismo, es necesario revisar las estrategias de mitigación del ruido del Aeropuerto El Dorado y evaluar seriamente las implicaciones en salud derivadas de su ampliación.

\section{Agradecimientos}

Agradecemos en forma especial al grupo de trabajo de la Línea Aire, Ruido y Radiaciones Electromagnéticas del Hospital Fontibón, así como a Angie Gómez, referente del área de medio ambiente, a Jhon Jairo Abella y a David Muñoz.

\section{Conflicto de intereses}

Los autores declaran no tener ningún tipo de conflicto de intereses.

\section{Financiación}

Todo el proyecto fue financiado por la Secretaría Distrital de Salud de Bogotá.

\section{Referencias}

1. Sierra JC, Jiménez C, Martín JD. Calidad del sueño en estudiantes universitarios: importancia de la higiene del sueño. Rev Salud Mental. 2002;25:35-44.

2. Muzet A. Environmental noise, sleep and health. Sleep Med Rev. 2007;11:135-42.

3. Quiroz L, Hernández LJ, Corredor J, Rico V, Rugeles C, Medina K. Efectos auditivos y neuropsicológicos por exposición a ruido ambiental en una localidad de Bogotá en 2010. Rev Salud Pública. 2013;15:116-28.

4. Londoño JL, Quinchía R, Restrepo H, Vieco F. Efectos auditivos y sicológicos del ruido producido por el tráfico aéreo del aeropuerto El Dorado en las poblaciones de Engativá y Fontibón. Fecha de consulta: 12 de septiembre de 2012. Disponible en: http://www.institutodeestudiosurbanos.info/ dmdocuments/cendocieu/coleccion_digital/Operacion_ Estrategia_Aeropuerto/Efectos_Auditivos_SicologicosLondono-J-2000-Info_Final.pdf.

5. Escobar FE, Eslava J. Validación colombiana del índice de calidad de sueño de Pittsburg. Rev Neurol. 2005;40:150-5.

6. Basner M, Grifahn B, van den Berg M. Aircraft noise effects on sleep: Mechanisms, mitigation and research needs. Noise Health. 2010;12:95-109. http://dx.doi. org/10.4103/1463-1741.63210

7. Kogan P. Análisis de la eficiencia de la ponderación "A" para evaluar efectos del ruido en el ser humano (tesis). Valdivia (Chile): Universidad Austral de Chile; 2004. p. 178.

8. Rueda M, Díaz LA, Osuna E. Definición, prevalencia y factores de riesgo de insomnio en la población general. Revista de la Facultad de Medicina. 2008;56:222-34.

9. Kawada T. Noise and health - sleep disturbance in adults. J Occup Health. 2011;53:413-6. http://dx.doi.org/10.1539/ joh.11-0071-RA 